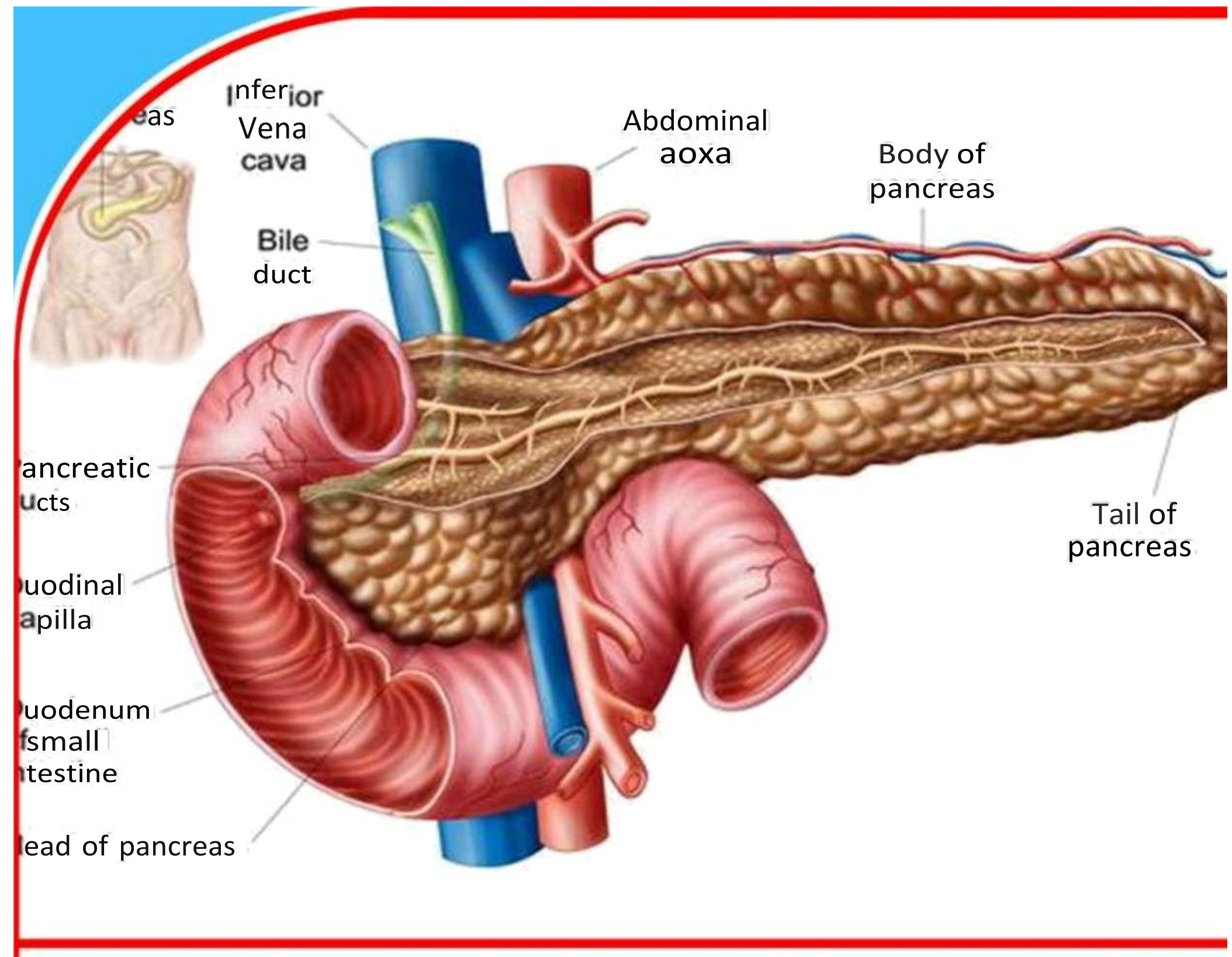

Ethnobotanical study of wild flora of Haroonabad, District Bahawalnagar, Punjab, Pakistan.

Zobia Anwer

Siddra Shabbir

Qurat-ul-ain

Tanzeela Iram

Sumaira Tariq

Hina Murad 


\title{
Ethnobotanical study of wild flora of Haroonabad, District Bahawalnagar, Punjab, Pakistan
}

\author{
Zobia Anwer ${ }^{1}$, Siddra Shabbir², Qurat-ul-ain², Tanzeela Iram², Sumaira Tariq ${ }^{2}$, Hina Murad² \\ ${ }^{1}$ Department of Botany, The Islamia University Bahawalpur, Pakistan \\ ${ }^{2}$ Department of Botany, University of Agriculture Faisalabad, Pakistan \\ Corresponding Author’s Email: toqeerfqw@gmail.com
}

\begin{abstract}
Purpose: The first purpose of this study to record the new and rare use of medicinal plants in the selected area; secondly to discover the plants which were unexplored in the past and thirdly to record the Ethnobotanical data of occurring plant species.

Methodology: The studied area surveyed from July to December. The plants were dried and mounted on standard herbarium sheets. The Ethnobotanical data were collected from 85 local people of Haroonabad by questionnaire.

Findings: The wild flora contained 81 species within 28 families. The largest family was Poaceae with 15 species followed by Euphorbiaceae with 8 species while Asteraceae and Amaranthaceae with 7 species. The life span of plants was comprised of 47 annual species (58\%) and 34 perennial species (42\%). The life-form spectrum explains that Therophyte 48 species (59\%) were the dominant followed by Phanerophytes 7 species (8\%) and Chaemophytes 13 species (17\%), Hemicryptophyte 8 species (10\%), Geophytes 3 species (4\%) Halophyte 1 specie $(1 \%)$ and Parasite 1 specie $(1 \%)$. Leaf venation classes of plants consisted of reticulate 28 species (34\%), pinnate 29 species (36\%), parallel 20 species (25\%), palmate 2 species $(2.5 \%)$ and 2 species $(2.5 \%)$ were leafless thus had no leaf venation. The plant species with herbaceous stems was 68 while with woody stems 13 species. The Ethnobotanical data of 70 species were recorded because 11 plants were not known by anyone. The range of UV value was 0.09 (Chrozophora plicata Vahl.) to 0.78 (Acacia nilotica L.) and the RCF value range was 0.03 (Imperata cylindrica L.) to 0.95 (Acacia nilotica L.). The range of ICF value was recorded from 0.45 (Hormonal disorder) to 0.78 (Respiratory disease). $57 \%$ whole plant followed by leaves $(53 \%)$ was used in the ayurvedic field. Therophytes were supported in the studied region for the reason that the region is a semi-arid zone of Punjab, Pakistan.
\end{abstract}

Unique contribution: The present comprehensive study provides a basic point for other researchers and enhances the knowledge of poor people related to ayurvedic field. The Ethnobotanical study tries to attract the attention towards the conservation strategies of wild plants.

Key words: Survey, Wild flora, Questionnaire, Side effects, Ethnobotanical study

Abbreviations: UV, Use Value; RCF, Relative Frequency Citation; ICF, Informant Consensus Factor 


\section{INTRODUCTION}

The Ethnobotanical study plays an important role in exposing the relationship between humans and plants [16]. The history of getting medication from the plant is very old. After fulfilling the basic needs like food, fuel shelter and man also uses plants to treat various ailments [13]. In the era we are living in today, $80 \%$ of the population is not able to get more expensive treatments. People get medicines from plants to cure their illnesses because herbal medicines are easily available and cheaper than allopathic medicines [1]. There is no doubt that the poor people around the world treat their diseases with herbal medicines. Some plants are specific to the treatment of only one disease and some plants are used to treat many ailments [17]. Ethnobotanical knowledge is now at risk because this knowledge is not transferring from the older generation to the younger generation [2]. The record of the flora of any area by plant taxonomist gain more importance around the world. It provides information about natural vegetation of specific areas [9]. Wild flora in the medicinal field is too much important because, it used in medicinal production and produces immunity in the body to fight against many diseases like cancer, diabetes and heart disease etc. [15]. The purpose of this research to inform the people of developing countries and native people of Haroonabad that wild plants are very important. These wild plants can be used to treat many ailments that are much cheaper than allopathic medicines and their side effects will be much less.

\section{MATERIALS AND METHODS}

The whole study area Haroonabad surveyed from July to December. The plants were collected after conducting 15 surveys of the selected area. The plant specimens collected from the area. The specimens were dried and mounted on standard herbarium sheets. The collected specimens were identified with the help of various monographs \{(http://www.ipni.org), (www.theplantlist.org) and (http://www.ars-grin.gov/cgi-bin/npgs/html/queries.pl)\}.

Climate of studied area: The climate in this area is very hot and harsh. Temperature fluctuations are also very high. In the summer, the mean temperature is between $35^{\circ} \mathrm{C}$ and $60^{\circ} \mathrm{C}$ and in winter the mean temperature is between $10^{\circ} \mathrm{C}$ and $20^{\circ} \mathrm{C}$. In the desert of Haroonabad, organic matter is very low so it is not considered good soil. The desert is changing into Death Valley due to high temperature, windstorms and a high rate of evaporation. May and June are the warmest months of this area. There are some plants in this area that come out of the ground after rain and die after producing seeds.

Collection of Ethnobotanical data: The total number of participants in the survey was 85 (Male: 51, Female: 34) and they were interviewed by questionnaire. All participants in the survey were between the ages of 37 and 63 years. The eleven percent (9) of the participants was from the homeopathic field and the rest were from different departments like labors, farming, teachers, trading and house-wife. 
European Journal of Biology

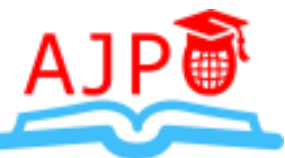

Vol.5, Issue 1, pp 41-62, 2020

www.ajpojournals.org

Table 1: Demographic data of participants

\begin{tabular}{lllll}
\hline Sr. No. & Variables & Categories & No. of persons & Percentage \\
\hline $\mathbf{1}$ & Gender & Male & 51 & 60 \\
$\mathbf{2}$ & \multirow{3}{*}{ Participant profession } & Female & 34 & 40 \\
& & Hakim & 9 & 11 \\
& & Labor & 17 & 20 \\
& & Farming & 15 & 18 \\
& & Teachers & 13 & 15 \\
& & Trading & 19 & 22 \\
$\mathbf{3}$ & Educational background & House-wife & 21 & 25 \\
& & Illiterate & 0 & 0 \\
& Middle & 31 & 37 \\
& & Matric & 17 & 20 \\
& & Intermediate & 15 & 18 \\
$\mathbf{4}$ & Bachelor & 8 & 9 \\
& \multirow{4}{*}{ Age } & Specialization & 14 & 16 \\
& & $30-40$ & 4 & 5 \\
& & $40-50$ & 35 & 41 \\
& & $50-60$ & 37 & 43 \\
& & 60 & 9 & 11 \\
\hline
\end{tabular}

Quantitative analysis of Ethnobotanical data

Use Value (UV): The UV was used to explore the importance of any plant species. According to the formula of Phillips et al. (1994), the UV was determined.

$$
\mathrm{UV}=\Sigma \mathrm{U} / \mathrm{n}
$$

$\mathrm{U}=$ No. of participants for a given species

$\mathrm{n}=$ Total number of participants

Relative Frequency Citation (RFC): According to Vitalini et al. (2013), RFC value was calculated

$$
\mathrm{RFC}=\mathrm{FC} / \mathrm{N}
$$

$\mathrm{FC}=$ Number of participants who points out the use of plant

$\mathrm{N}=$ Total number of participants

Informant Consensus Factor (ICF): According to Heinrich et al. (1998), ICF was calculated

$$
\mathrm{ICF}=\{(\mathrm{Nur}-\mathrm{Nt}) / \mathrm{Nur}-1\}
$$

Nur $=$ Total number of use informants mentioned for a particular disease category

$\mathrm{Nt}=$ Total number of plant species used for a particular disease category 
European Journal of Biology

Vol.5, Issue 1, pp 41-62, 2020

www.ajpojournals.org

\section{RESULTS}

The present study examined the wild flora of Haroonabad, District Bahawalnagar which indicates that the wild flora of this area belongs to 71 species of 28 families. Along with these presented 69 herbs (85\%), 5 trees (6\%) and 7 shrubs (9\%) species by growth habit (Table 4$)$. The most commonly represented family was Poaceae (15 species) followed by Euphorbiaceae (8 species), Asteraceae (7 species) and Amaranthaceae (7 species). Haloxylon salicornicum L. and Cuscuta campestris Yunk. Were leafless plant species. Annual plants (58\%) were more prominent than perennial plants (42\%). The studied region supported the Therophytes (59\%) and the majority of plants contained the pinnate leaf venation (36\%).

The ethnobotanists of Azad Jammu and Kashmir District Kotli collected the 463 plant species belonging to 306 genera and 93 families and reported that the Poaceae family was the dominant among all families [11]. In Pakistan Tolipir National Park, 35 tree species, 19 shrubs, 3 epiphytes, 4 climbers, 75 herbs, 10 ferns, 1 moss and 1 lichen species were recorded. The herbs were dominant in the studied region. The herbs have more importance in the medicinal field due to their high reforestation capacity [5].

Table 3: List of wild flora in Haroonabad, District Bahawalnagar, Pakistan

\begin{tabular}{|c|c|c|c|c|c|c|c|c|}
\hline $\begin{array}{l}\text { Sr. } \\
\text { No. }\end{array}$ & Scientific name & $\begin{array}{l}\text { Common } \\
\text { name }\end{array}$ & Family & $\mathbf{L V}$ & LFS & ST & $\mathbf{L S}$ & $\overline{\text { GH }}$ \\
\hline 1 & $\begin{array}{l}\text { Trianthema } \\
\text { portulacastrum } \mathrm{L} \text {. }\end{array}$ & $\begin{array}{l}\text { Black } \\
\text { pigweed }\end{array}$ & Aizoaceae & PIN & $\mathrm{Th}$ & $\mathrm{Hr}$ & $\mathrm{A}$ & $\mathrm{H}$ \\
\hline 2 & Zaleya pentendra $\mathrm{L}$. & Biskhapra & Aizoaceae & PIN & $\mathrm{Th}$ & $\mathrm{Hr}$ & $\mathrm{P}$ & $\mathrm{H}$ \\
\hline 3 & Achyranthes aspera L. & Devil weed & Amaranthaceae & RCL & $\mathrm{Th}$ & $\mathrm{W}$ & $\mathrm{P}$ & $\mathrm{H}$ \\
\hline 4 & Alternanthera sessilis L. & Gandal booti & Amaranthaceae & PIN & $\mathrm{Ch}$ & $\mathrm{Hr}$ & $\mathrm{P}$ & $\mathrm{H}$ \\
\hline 5 & Amaranthus viridis L. & jungle cholai & Amaranthaceae & PIN & $\mathrm{Th}$ & $\mathrm{Hr}$ & A & $\mathrm{H}$ \\
\hline 6 & $\begin{array}{l}\text { Aerva javanica (Burm. } \\
\text { f.) Schult. }\end{array}$ & Bui & Amaranthaceae & PIN & $\mathrm{Ch}$ & $\mathrm{Hr}$ & $\mathrm{P}$ & $\mathrm{H}$ \\
\hline 7 & Digera muricata L. & Tandla & Amarantheceae & PIN & $\mathrm{Ch}$ & $\mathrm{Hr}$ & A & $\mathrm{H}$ \\
\hline 8 & $\begin{array}{l}\text { Haloxylon salicornicum } \\
\text { L. }\end{array}$ & Lana & Amarantheceae & LL & $\mathrm{Ch}$ & $\mathrm{W}$ & $\mathrm{P}$ & $S$ \\
\hline 9 & $\begin{array}{l}\text { Suaeda fruticosa (L.) } \\
\text { Forsk }\end{array}$ & Kali lani & Amaranthaceae & RCL & $\mathrm{Ha}$ & $\mathrm{W}$ & $\mathrm{P}$ & $S$ \\
\hline
\end{tabular}

Contin......

\begin{tabular}{|c|c|c|c|c|c|c|c|c|}
\hline $\begin{array}{l}\text { Sr. } \\
\text { No. }\end{array}$ & Scientific name & $\begin{array}{l}\text { Common } \\
\text { name }\end{array}$ & Family & $\mathbf{L V}$ & LFS & ST & $\mathbf{L S}$ & $\overline{\text { GH }}$ \\
\hline 10 & $\begin{array}{l}\text { Calotropis procera } \\
\text { (Aiton) }\end{array}$ & Aak & Asclepiadaceae & PIN & $\mathrm{Ch}$ & $\mathrm{W}$ & $\mathrm{P}$ & $\bar{S}$ \\
\hline 11 & Cichorium intybus L. & Kasni & Asteraceae & PIN & Th & $\mathrm{Hr}$ & A & $\mathrm{H}$ \\
\hline 12 & Cirsium arvense L. & Leh & Asteraceae & PIN & Th & $\mathrm{Hr}$ & $\mathrm{P}$ & $\mathrm{H}$ \\
\hline 13 & Conyza ambigua $\mathrm{L}$. & Horseweed & Asteraceae & RCL & Th & $\mathrm{Hr}$ & A & $\mathrm{S}$ \\
\hline 14 & Eclipta alba $\mathrm{L}$. & False daisy & Asteraceae & RCL & $\mathrm{Ch}$ & $\mathrm{Hr}$ & $\mathrm{P}$ & $\mathrm{H}$ \\
\hline 15 & Parthenium & Chatak & Asteraceae & PIN & $\mathrm{Th}$ & $\mathrm{Hr}$ & A & $\mathrm{H}$ \\
\hline
\end{tabular}




\section{www.ajpojournals.org}

\begin{tabular}{|c|c|c|c|c|c|c|c|c|}
\hline & hysterophorus L. & chandni & & & & & & \\
\hline 16 & Sonchus asper L. & $\begin{array}{l}\text { Spiny sow } \\
\text { thistle }\end{array}$ & Asteraceae & PIN & $\mathrm{Th}$ & $\mathrm{Hr}$ & A & $\mathrm{H}$ \\
\hline 17 & Sonchus oleraceus L. & $\begin{array}{l}\text { Smooth sow } \\
\text { thistle }\end{array}$ & Asteraceae & RCL & $\mathrm{Th}$ & $\mathrm{Hr}$ & $\mathrm{A}$ & $\mathrm{H}$ \\
\hline 18 & $\begin{array}{l}\text { Cordia dichotoma } \mathrm{G} \text {. } \\
\text { Forst }\end{array}$ & Lasura & Boraginaceae & RCL & $\mathrm{Ph}$ & $\mathrm{W}$ & $\mathrm{P}$ & $\mathrm{T}$ \\
\hline 19 & Heliotropium indicum $\mathrm{L}$. & Oont chara & Boraginaceae & PIN & $\mathrm{Th}$ & $\mathrm{Hr}$ & $\mathrm{A}$ & $\mathrm{H}$ \\
\hline 20 & Sisymbrium irio L. & Jangli sarson & Brassicaceae & RCL & $\mathrm{Th}$ & $\mathrm{Hr}$ & $\mathrm{A}$ & $\mathrm{H}$ \\
\hline 21 & Cleome viscose L. & Hulhul & Capparidaceae & RCL & $\mathrm{Th}$ & $\mathrm{Hr}$ & A & $\mathrm{H}$ \\
\hline 22 & Spergula arvensis L. & Jangli dhania & Caryophyllaceae & RCL & $\mathrm{Th}$ & $\mathrm{W}$ & $\mathrm{A}$ & $\mathrm{H}$ \\
\hline 23 & $\begin{array}{l}\text { Chenopodium } \\
\text { ambrosides } \mathrm{L} \text {. }\end{array}$ & Worm seed & Chenopodiaceae & RCL & Th & $\mathrm{Hr}$ & $\mathrm{A}$ & $\mathrm{H}$ \\
\hline 24 & Chenopodium album $\mathrm{L}$. & Bathu & Chenopodiaceae & PLM & $\mathrm{Th}$ & $\mathrm{Hr}$ & $\mathrm{A}$ & $\mathrm{H}$ \\
\hline 25 & $\begin{array}{l}\text { Chenopodium } \\
\text { berlandieri Moq. }\end{array}$ & Jangli bathu & Chenopodiaceae & RCL & Th & $\mathrm{Hr}$ & $\mathrm{A}$ & $\mathrm{H}$ \\
\hline 26 & Chenopodium murale L. & Krund & Chenopodiaceae & RCL & $\mathrm{Th}$ & $\mathrm{Hr}$ & A & $\mathrm{H}$ \\
\hline 27 & Convolvulus arvensis $\mathrm{L}$. & Lehli, baily & Convolvulaceae & PIN & $\mathrm{Th}$ & $\mathrm{Hr}$ & $\mathrm{P}$ & $\mathrm{H}$ \\
\hline 28 & $\begin{array}{l}\text { Convolvulus } \\
\text { Pluricaulis Choisy. }\end{array}$ & Makro & Convolvulaceae & PIN & $\mathrm{Th}$ & $\mathrm{Hr}$ & $\mathrm{P}$ & $\mathrm{H}$ \\
\hline 29 & $\begin{array}{l}\text { Citrulus } \\
\text { Colocynthis L. }\end{array}$ & Kor tumma & Cucurbitaceae & PIN & $\mathrm{He}$ & $\mathrm{W}$ & $\mathrm{A}$ & $\mathrm{H}$ \\
\hline 30 & Cucumis melo L. & Musk-melon & Cucurbitaceae & RCL & $\mathrm{T}$ & $\mathrm{Hr}$ & $\mathrm{A}$ & $\mathrm{H}$ \\
\hline 31 & $\begin{array}{l}\text { Cuscuta campestris } \\
\text { Yunk. }\end{array}$ & Amar bale & Cuscutaceae & LL & $\mathrm{Pa}$ & $\mathrm{Hr}$ & $\mathrm{A}$ & $\mathrm{H}$ \\
\hline 32 & Cyperus rotundus $\mathrm{L}$. & $\begin{array}{l}\text { Chotibhoin, } \\
\text { kalooro }\end{array}$ & Cyperaceae & PAR & $\mathrm{Th}$ & $\mathrm{Hr}$ & $\mathrm{P}$ & $\mathrm{H}$ \\
\hline 33 & $\begin{array}{l}\text { Fimbristylis dichotoma } \\
\text { L. }\end{array}$ & Coco grass & Cyperaceae & PAR & $\mathrm{He}$ & $\mathrm{Hr}$ & $\mathrm{P}$ & $\mathrm{H}$ \\
\hline
\end{tabular}

Contin......

\begin{tabular}{|c|c|c|c|c|c|c|c|c|}
\hline $\begin{array}{l}\text { Sr. } \\
\text { No. }\end{array}$ & Scientific name & $\begin{array}{l}\text { Common } \\
\text { name }\end{array}$ & Family & $\mathbf{L V}$ & LFS & ST & $\mathbf{L S}$ & $\overline{\text { GH }}$ \\
\hline 34 & $\begin{array}{l}\text { Schoenoplectus } \\
\text { mucronatus L. }\end{array}$ & Rush booti, & Cyperaceae & PAR & $\mathrm{Th}$ & $\mathrm{Hr}$ & $\mathrm{P}$ & $\mathrm{H}$ \\
\hline 35 & $\begin{array}{l}\text { Chrozophora } \\
\text { Plicata Vahl. }\end{array}$ & Giradol & Euphorbiaceae & RCL & $\mathrm{Ch}$ & $\mathrm{Hr}$ & A & $\mathrm{H}$ \\
\hline 36 & $\begin{array}{l}\text { Euphorbia granulate } \\
\text { Orteg. }\end{array}$ & $\begin{array}{l}\text { Hazar dani } \\
\text { dodhak }\end{array}$ & Euphorbiaceae & PAR & $\mathrm{Th}$ & $\mathrm{Hr}$ & A & $\mathrm{H}$ \\
\hline 37 & $\begin{array}{l}\text { Euphorbia helioscopia } \\
\text { L. }\end{array}$ & $\begin{array}{l}\text { Chhatri } \\
\text { dodhak }\end{array}$ & Euphorbiaceae & PIN & $\mathrm{Th}$ & $\mathrm{Hr}$ & A & $\mathrm{H}$ \\
\hline 38 & Euphorbia hirta L. & Laldodhak & Euphorbiaceae & RCL & $\mathrm{Th}$ & $\mathrm{Hr}$ & A & $\mathrm{H}$ \\
\hline 39 & $\begin{array}{l}\text { Euphorbia microphylla } \\
\text { Heyne ex. Roth. }\end{array}$ & $\begin{array}{l}\text { Nani dudheli, } \\
\text { sandmat }\end{array}$ & Euphorbiaceae & RCL & $\mathrm{Th}$ & $\mathrm{Hr}$ & A & $\mathrm{H}$ \\
\hline 40 & Euphorbia thymifolia L. & Gulf sandmat & Euphorbiaceae & RCL & Th & $\mathrm{Hr}$ & A & $\mathrm{H}$ \\
\hline
\end{tabular}




\begin{tabular}{|c|c|c|c|c|c|c|c|c|}
\hline 41 & $\begin{array}{l}\text { Phyllanthus } \\
\text { maderaspatensis L. }\end{array}$ & Kanocha & Euphorbiaceae & PIN & $\mathrm{Ch}$ & $\mathrm{Hr}$ & $\bar{A}$ & $\bar{H}$ \\
\hline 42 & Ricinus communis $\mathrm{L}$. & Arind & Euphorbiaceae & PLM & $\mathrm{Ph}$ & $\mathrm{W}$ & $\mathrm{P}$ & S \\
\hline 43 & Acacia karoo Hayne. & Pahari keekar & Fabaceae & PIN & $\mathrm{Ph}$ & $\mathrm{W}$ & $\mathrm{P}$ & $S$ \\
\hline 44 & Accaia nilotica $\mathrm{L}$. & Keekar & Fabaceae & PIN & $\mathrm{Ph}$ & $\mathrm{W}$ & $\mathrm{P}$ & $\mathrm{T}$ \\
\hline 45 & Albizia lebbek L. & Sharin & Fabaceae & RCL & $\mathrm{Ph}$ & $\mathrm{W}$ & $\mathrm{P}$ & $\mathrm{T}$ \\
\hline 46 & Cassia fistula $\mathrm{L}$. & Amaltas & Fabaceae & RCL & $\mathrm{Ph}$ & $\mathrm{W}$ & $\mathrm{P}$ & $\mathrm{T}$ \\
\hline 47 & $\begin{array}{l}\text { Hydrilla verticillata } \\
\text { (L.f.) Royle }\end{array}$ & Jala & $\begin{array}{l}\text { Hydrochlorit- } \\
\text { aceae }\end{array}$ & PAR & Th & $\mathrm{Hr}$ & $\mathrm{P}$ & $\mathrm{H}$ \\
\hline 48 & $\begin{array}{l}\text { Ocimum } \\
\text { Basilicum L. }\end{array}$ & Niazboo & Limiacae & PIN & $\mathrm{Ch}$ & $\mathrm{Hr}$ & $\mathrm{A}$ & $\mathrm{H}$ \\
\hline 49 & Oxalis corniculata $\mathrm{L}$. & Khati boti & Oxalidaceae & PIN & Th & $\mathrm{Hr}$ & A & $\mathrm{H}$ \\
\hline 50 & Lathyrus aphaca L. & Jangli matar & Papilionaceae & RCL & Th & $\mathrm{Hr}$ & A & $\mathrm{H}$ \\
\hline 51 & $\begin{array}{l}\text { Medicago polymorpha } \\
\text { L. }\end{array}$ & Maina & Papilionaceae & PIN & Th & $\mathrm{Hr}$ & $\mathrm{A}$ & $\mathrm{H}$ \\
\hline 52 & Pongamia pinnata $\mathrm{L}$. & Sukhchain & Paplionaceae & PIN & $\mathrm{Ph}$ & $\mathrm{W}$ & $\mathrm{P}$ & $\mathrm{T}$ \\
\hline 53 & Vicia sativa $\mathrm{L}$. & Revari & Paplionaceae & PIN & Th & $\mathrm{Hr}$ & $\mathrm{A}$ & $\mathrm{H}$ \\
\hline 54 & Phyllanthus niruri L. & $\begin{array}{l}\text { Gulf leaf } \\
\text { flower }\end{array}$ & Phyllanthaceae & PIN & $\mathrm{Ch}$ & $\mathrm{Hr}$ & A & $\mathrm{H}$ \\
\hline 55 & Avena fatua $\mathrm{L}$. & Javi & Poaceae & PAR & Th & $\mathrm{Hr}$ & A & $\mathrm{H}$ \\
\hline 56 & $\begin{array}{l}\text { Bromus catharticus } \\
\text { Vahl. }\end{array}$ & Chawli ghass & Poaceae & PAR & $\mathrm{He}$ & $\mathrm{Hr}$ & $\mathrm{P}$ & $\mathrm{H}$ \\
\hline 57 & Brachiaria ramose $\mathrm{L}$. & Sudan ghass & Poaceae & PAR & Th & $\mathrm{Hr}$ & $\mathrm{A}$ & $\mathrm{H}$ \\
\hline 58 & Cenchrus ciliaris $\mathrm{L}$. & Dhamasa & Poaceae & PAR & $\mathrm{He}$ & $\mathrm{Hr}$ & $\mathrm{P}$ & $\mathrm{H}$ \\
\hline 59 & Cyanodon dactylon $\mathrm{L}$. & Khabal ghass & Poaceae & PAR & $\mathrm{He}$ & $\mathrm{Hr}$ & $\mathrm{P}$ & $\mathrm{H}$ \\
\hline 60 & $\begin{array}{l}\text { Dactyloctenum } \\
\text { aegyptium } \mathrm{L} .\end{array}$ & $\begin{array}{l}\text { Madhana } \\
\text { ghass }\end{array}$ & Poaceae & PAR & Th & $\mathrm{Hr}$ & A & $\mathrm{H}$ \\
\hline
\end{tabular}

Contin......

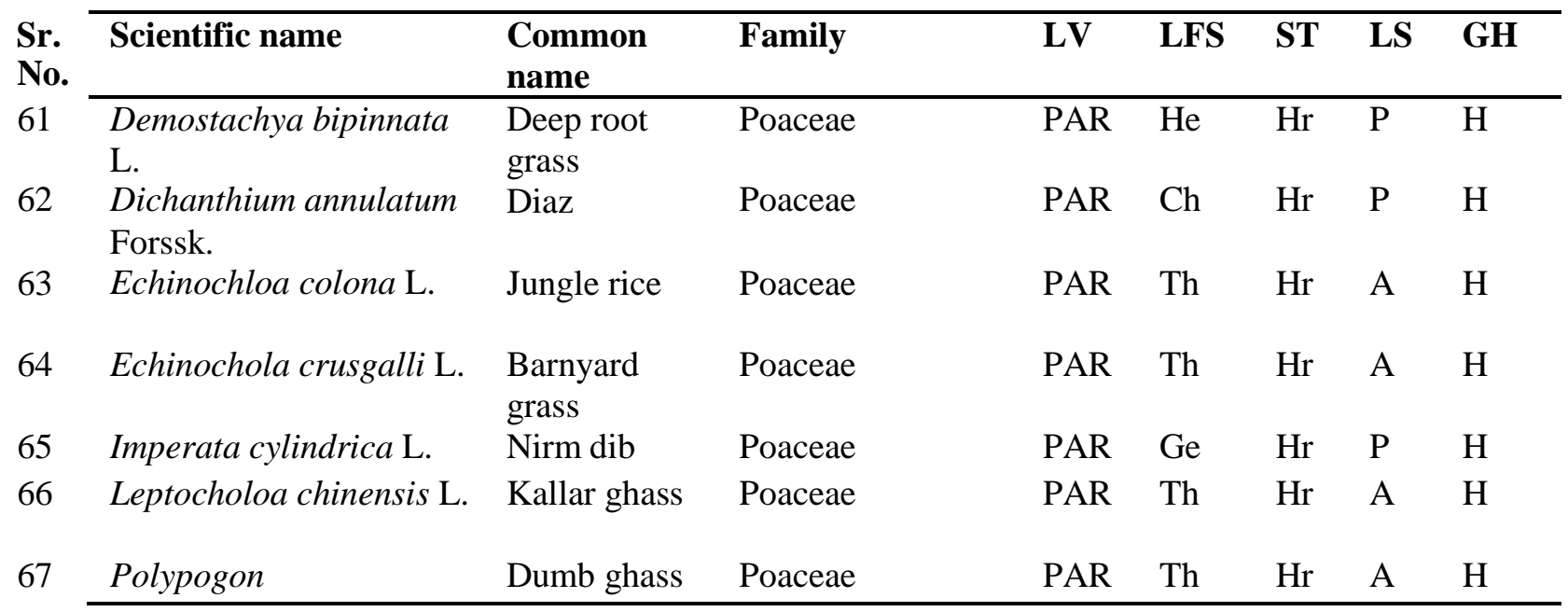




\begin{tabular}{|c|c|c|c|c|c|c|c|c|}
\hline & monspeliensis L. & & & & & & & \\
\hline 68 & $\begin{array}{l}\text { Sachharum benghalense } \\
\text { Retz. }\end{array}$ & $\begin{array}{l}\text { Sarkanda, } \\
\text { munj }\end{array}$ & Poaceae & PAR & $\mathrm{He}$ & $\mathrm{Hr}$ & $\mathrm{P}$ & $\mathrm{H}$ \\
\hline 69 & Sorghum halepense L. & Baru & Poaceae & PAR & $\mathrm{He}$ & $\mathrm{Hr}$ & $\mathrm{P}$ & $\mathrm{H}$ \\
\hline 70 & Emex spinosa L. & $\begin{array}{l}\text { Trkandi } \\
\text { palak }\end{array}$ & Polygonaceae & RCL & Th & $\mathrm{Hr}$ & A & $\mathrm{H}$ \\
\hline 71 & Rumex dentatus L. & Jangli palak & Polygonaceae & RCL & $\mathrm{Ge}$ & $\mathrm{Hr}$ & A & $\mathrm{H}$ \\
\hline 72 & Portulaca oleracea L. & Qulfa, Lonak & Portulaceae & $\mathrm{RCL}$ & $\mathrm{Th}$ & $\mathrm{Hr}$ & A & $\mathrm{H}$ \\
\hline 73 & Anagallis arvensis L. & Billi booti & Primulaceae & RCL & Th & $\mathrm{Hr}$ & A & $\mathrm{H}$ \\
\hline 74 & Renunculus muricatus L. & Ghorr summi & Renunculaceae & RCL & $\mathrm{Ge}$ & $\mathrm{Hr}$ & $\mathrm{P}$ & $\mathrm{H}$ \\
\hline 75 & Dhatura alba $\mathrm{L}$. & Jimson weed & Solanaceae & $\mathrm{RCL}$ & $\mathrm{Th}$ & $\mathrm{Hr}$ & $\mathrm{P}$ & $\mathrm{H}$ \\
\hline 76 & Physalis minima $\mathrm{L}$. & Rasbari & Solanaceae & RCL & $\mathrm{Ch}$ & $\mathrm{Hr}$ & A & $\mathrm{H}$ \\
\hline 77 & Solanum nigrum L. & Mako, Peelak & Solanaceae & RCL & $\mathrm{Th}$ & $\mathrm{Hr}$ & A & $\mathrm{H}$ \\
\hline 78 & Withania somnifera $\mathrm{L}$. & Aksin & Solanaceae & PIN & $\mathrm{Ch}$ & $\mathrm{Hr}$ & $\mathrm{P}$ & $\mathrm{S}$ \\
\hline 79 & $\begin{array}{l}\text { Sphenoclea zeylanica } \\
\text { Gaertn. }\end{array}$ & Mirch booti & Sphenocolaceae & PIN & Th & $\mathrm{Hr}$ & A & $\mathrm{H}$ \\
\hline 80 & Corchorus tridens L. & Jangli patsan & Tilaceae & PIN & Th & $\mathrm{Hr}$ & A & $\mathrm{H}$ \\
\hline 81 & Phyla nodiflora L. & Bukkan-booti & Verbenaceae & PIN & $\mathrm{Th}$ & $\mathrm{Hr}$ & $\mathrm{P}$ & $\mathrm{H}$ \\
\hline
\end{tabular}

Legends = LV; Leaf Venation, PIN; Pinnate, RCL; Reticulate, LL; Leafless, PAR; Parallel, PLM; Palmate, LFS; Life Form Spectrum, Th; Therophyte, Ph; Phanerophytes, Ge; Geophytes, Ch; Chaemophytes, Pa; Parasite, He; Hemicryptophytes, ST; Stem type, LS; Life Span, GH; Growth Habitat, Hr; Herbacium, W; Woody, A; Annual, P; Perennial, H; Herb, S; Shurb, T; Tree. 
European Journal of Biology

Vol.5, Issue 1, pp 41-62, 2020

www.ajpojournals.org

Table 4: Showing the number and percentage of different parameters

\begin{tabular}{|c|c|c|c|c|c|c|}
\hline \multicolumn{7}{|c|}{ Life span } \\
\hline \multicolumn{3}{|c|}{$\begin{array}{l}\text { No. of } \\
\text { Species }\end{array}$} & Percentage & \multicolumn{2}{|r|}{$\begin{array}{l}\text { No. of } \\
\text { Species }\end{array}$} & Percentage \\
\hline Annual & 47 & & 58 & Perennial & 34 & 42 \\
\hline \multicolumn{7}{|c|}{ Life form spectrum } \\
\hline & & $\begin{array}{l}\text { No. of } \\
\text { Species }\end{array}$ & Percentage & & $\begin{array}{l}\text { No. of } \\
\text { Species }\end{array}$ & Percentage \\
\hline Phanerophytes & & 7 & 8 & Chaemophytes & 13 & 17 \\
\hline Geophytes & & 3 & 3 & Therophytes & 48 & 59 \\
\hline Hemicryptophy & ytes & 8 & 10 & Parasite & 1 & 1 \\
\hline
\end{tabular}

Growth habitat

\begin{tabular}{llllllll}
\hline & $\begin{array}{l}\text { No. of } \\
\text { Species }\end{array}$ & Percentage & $\begin{array}{l}\text { No. of } \\
\text { Species }\end{array}$ & Percentage & $\begin{array}{l}\text { No. of } \\
\text { Species }\end{array}$ & Percentage \\
\hline Herbs & 69 & 85 & Shrubs & 7 & 9 & Tree & 5 \\
\hline
\end{tabular}

Leaf venation

\begin{tabular}{lll|lll}
\hline & No. of Species & Percentage & & No. of Species & Percentage \\
\hline Pinnate & 29 & 36 & Reticulate & 28 & 34 \\
\cline { 3 - 4 } Parallel & 20 & 25 & Palmate & 2 & 2.5 \\
\hline
\end{tabular}

Stem type

\begin{tabular}{lll|lll}
\hline & No. of Species & Percentage & & No. of Species & Percentage \\
\hline Herbaceous & 68 & 84 & Woody & 13 & 16 \\
\hline
\end{tabular}




\section{Ethnobotanical data}

The Ethnobotanical data of 81 plants were collected from 85 participants through a questionnaire. No one knew the use of 11 plants (Vicia sativa L., Medicago polymorpha L., Leptocholoa Chinese L., Emox spinosa L., Sorghum halepense L., Cascuta campestris Yunk., Schoenoplectus mucronatus L., Phyllanthus maderaspatensis L., Corchorus tridens L., Dichanthium annulatum Forsk. and Bromus catharticus Vahl.) out of 81. The range of UV value was 0.09 (Chrozophora plicata Vahl.) to 0.78 (Acacia nilotica L.) and RCF value range was 0.03 (Imperata cylindrica L.) to 0.95 (Acacia nilotica L.). The highest UV value means a lot of people were used this plant for treatment of ailments. The highest value of RCF means that a lot of people were declared that this (given) plant was useful in ayurvedic field. Residents of the studied area use various processes like juice, infusion, ash, extract, decoction, paste, tea, powder and poultice etc. to prepare a recipe for the cure of different illnesses. While making of decoction, Plant parts were boiled in water until the volume of water reduced to $1 / 4$ of original volume. Crude extract was gained by crushing and squeezing of plant parts. Some people were crushed the plant parts and smell it for the treatment of different diseases. Infusion in the ayurvedic field was considered good because bioactive components in plants were not degraded [4]. The majority of recipes were prepared from fresh plant parts [10]. The informants thought that the flowering period was best for plant collection because a lot of bioactive compounds were activated at this stage. The use of seeds and roots in the medicinal field is responsible for the extinction of plants [6].

Informants were aware of the side effects of wild plants as well as their benefits. Trianthema portulacastrum L. leaves were also used in a salad but a high dose of leaves also caused the paralysis. Amaranthus viridis L. was not toxic itself but if they grow in nitrogenous soil then it showed the toxic effects on consumers. The high dose of Calotropis gigantean (L.) W.T.Aiton slowed down the heartbeat and responsible for vomiting. Conyza ambigua L. caused the skin allergy after touching the plant. Parthenium hysterophorus L. also known as toxic wild plants with few benefits. It disturbed the respiratory system of consumers. The high dose of seeds of Cleome viscosa L. enhanced the stomach problem. The high concentration of oxalic acid Chenopodium berlandieri Moq. Produced the kidney stone. The high dose of Convolvulus Pluricaulis Choisy. Suddenly reduced the blood pressure. According to the informants, great caution was needed in using Citrullus Colocynthis L. as even a small dose of it caused the kidney problem, stomach disease and even death. The seed coat of Ricinus communis L. contained the poisonous compounds which caused the stomach ailments and high dose of oil can caused the death. The Acacia nilotica L. also needed a lot of caution in using it. It also had some side effects. If it used indiscriminately, it can be caused liver and stomach diseases. The un-ripened fruit of Solanum nigrum $L$. also showed the toxic effect.

According to researchers, the plants of Euphorbiaceae, Fabaceae, Asteraceae and Apocynaceae were considered toxic because majority of plants was contained a saponins, glycosides, steroids, alcohol, cyanogenic glycosides, resins and selenium etc. [3]. Herbs or wild plants were not beneficial or toxic. The use of plants made them beneficial or toxic. The dose of medicines varied from child to old. Dose was taken thrice or twice a day depending upon the 
European Journal of Biology

Vol.5, Issue 1, pp 41-62, 2020

www.ajpojournals.org

nature or situation of patients. The people used the inappropriate techniques for the preparation of medicines which showed the toxic or side effects on the health of consumers [12].

Table 5: Ethnobotanical data of wild flora of selected area

\begin{tabular}{|c|c|c|c|c|}
\hline Species name & Part used & Recipes with ailments & $\mathbf{U V}$ & RFC \\
\hline $\begin{array}{l}\text { Trianthema } \\
\text { portulacastrum } \mathrm{L} .\end{array}$ & $\begin{array}{l}\text { Leaves, } \\
\text { Roots, } \\
\text { Whole plant }\end{array}$ & $\begin{array}{l}\text { Leaves } \\
\text { *Powder and fresh leaves (odema, dropsy and } \\
\text { jaundice) } \\
\text { Roots } \\
\text { *Powder (liver, asthma and veneral discharge) } \\
\text { Whole plant } \\
\text { *Powder (alcoholic poisoning, veneral } \\
\text { discharge, heart disease and piles) }\end{array}$ & 0.35 & 0.13 \\
\hline $\begin{array}{l}\text { Zaleya pentendra } \\
\text { L. }\end{array}$ & $\begin{array}{l}\text { Leaves, } \\
\text { Whole plant }\end{array}$ & $\begin{array}{l}\text { Leaves } \\
\text { *Juice (stomach problem) } \\
\text { Whole plant } \\
\text { *Powder (urinary infection) }\end{array}$ & 0.23 & 0.09 \\
\hline $\begin{array}{l}\text { Achyranthes aspera } \\
\text { L. }\end{array}$ & $\begin{array}{l}\text { Seeds, } \\
\text { Flowers, } \\
\text { Roots, } \\
\text { Whole plant }\end{array}$ & $\begin{array}{l}\text { Seeds } \\
\text { *Snake bite } \\
\text { Flowers } \\
\text { *Rubbed with sugar and made medicine (dog } \\
\text { bite) } \\
\text { Roots } \\
\text { *Powder (stomach, cholera) } \\
\text { Leaves } \\
\text { *Paste (scorpion bite, insect sting paralysis } \\
\text { and nervous system disorder) } \\
\text { Whole plant } \\
\text { *Powder (washing soda, malaria and cleaning } \\
\text { teeth) } \\
\text { *Extract (diarrhea, dysentery and skin } \\
\text { diseases) }\end{array}$ & 0.31 & 0.07 \\
\hline $\begin{array}{l}\text { Alternanthera } \\
\text { sessilis L. }\end{array}$ & $\begin{array}{l}\text { Roots, } \\
\text { Whole plant }\end{array}$ & $\begin{array}{l}\text { Roots } \\
\text { *Juice (fever, cold, cough and blood } \\
\text { dysentery) } \\
\text { Whole plant } \\
\text { *Paste (wounds, draw out spines from body } \\
\text { and cooling agent) } \\
\text { *Baked with corn flour (menstrual disorder } \\
\text { and stomach disease) } \\
\text { *Dry plant mixed with salt (Stop excess } \\
\text { bleeding and blood vomiting) }\end{array}$ & 0.51 & 0.23 \\
\hline
\end{tabular}


European Journal of Biology

ISSN 2520-4738 (Online)

Vol.5, Issue 1, pp 41-62, 2020

www.ajpojournals.org

\begin{tabular}{lllcc}
\hline $\begin{array}{l}\text { Spergula arvensis } \\
\text { L. }\end{array}$ & Seeds & *Making bread & 0.14 & 0.16 \\
$\begin{array}{l}\text { Chenopodium } \\
\text { ambrosides } \mathrm{L} .\end{array}$ & Whole plant & $*$ Tea (inflammatory and lung infection) & 0.16 & 0.09 \\
\hline
\end{tabular}

Contin......

\begin{tabular}{|c|c|c|c|c|}
\hline Species name & Part used & Recipes with ailments & $\mathbf{U V}$ & RFC \\
\hline $\begin{array}{l}\text { Amaranthus viridis } \\
\mathrm{L} \text {. }\end{array}$ & $\begin{array}{l}\text { Leaves, } \\
\text { Roots, } \\
\text { Whole plant }\end{array}$ & $\begin{array}{l}\text { Leaves } \\
\text { *Juice (heart disease and eye wash to prevent } \\
\text { infections) } \\
\text { *Powder (inflammation) } \\
\text { Roots } \\
\text { *Juice (inflammation of urinary bladder, } \\
\text { constipation and dysentery) } \\
\text { Whole plant } \\
\text { *Powder (soap making) }\end{array}$ & 0.33 & 0.28 \\
\hline $\begin{array}{l}\text { Aerva javanica } \\
\text { (Burm.f.) Schult. }\end{array}$ & $\begin{array}{l}\text { Seeds, } \\
\text { Flowers, } \\
\text { Roots, } \\
\text { Leaves }\end{array}$ & $\begin{array}{l}\text { Seeds } \\
\text { *Boiled seeds (mouth disease) } \\
\text { Flowers } \\
\text { *Dry and fresh are used to stop the bleeding } \\
\text { and repair the damaged cell. } \\
\text { Roots } \\
\text { *Juice (eye washing) } \\
\text { Leaves } \\
\text { *Paste (inflammation) }\end{array}$ & & \\
\hline Digera Arvensis L. & $\begin{array}{l}\text { Stems, } \\
\text { Seeds, } \\
\text { Flowers }\end{array}$ & $\begin{array}{l}\text { Stems } \\
\text { *Chewing of stem improve the digestive } \\
\text { system } \\
\text { Seeds } \\
\text { *Urinary disorder } \\
\text { Flowers } \\
\text { *Dry (Urinary disease) }\end{array}$ & 0.15 & 0.19 \\
\hline $\begin{array}{l}\text { Haloxylon } \\
\text { salicornicum }\end{array}$ & Leaves & $\begin{array}{l}\text { Leaves } \\
\text { *Tea (minimize the pregnancy pain) } \\
\text { *Fresh juice (eye infection) } \\
\text { Whole plant } \\
\text { *Ash of whole plant (wounds) }\end{array}$ & 0.17 & 0.21 \\
\hline $\begin{array}{l}\text { Suaeda fruticosa } \\
\text { (L.) Forsk }\end{array}$ & Leaves & $\begin{array}{l}\text { Leaves } \\
\text { *Paste (ringworm infection and skin allergy) }\end{array}$ & 0.19 & 0.16 \\
\hline $\begin{array}{l}\text { Calotropis } \\
\text { gigantean }\end{array}$ & $\begin{array}{l}\text { Barks, Root, } \\
\text { Leaves }\end{array}$ & $\begin{array}{l}\text { Barks } \\
\text { *Smoke is inhaled (respiratory disease and }\end{array}$ & 0.42 & 0.56 \\
\hline
\end{tabular}


European Journal of Biology

ISSN 2520-4738 (Online)

Vol.5, Issue 1, pp 41-62, 2020

www.ajpojournals.org

W.T.Aiton

asthma)

*Dry powder mix in water (stomach and heart

problem

Roots

*Ash of dry roots (skin allergy)

Leaves

*Leaf juice rubbed on scorpion sting

Contin......

\begin{tabular}{|c|c|c|c|c|}
\hline Species name & Part used & Recipes with ailments & $\mathbf{U V}$ & RFC \\
\hline $\begin{array}{l}\text { Cichorium intybus } \\
\text { L. }\end{array}$ & $\begin{array}{l}\text { Leaves, } \\
\text { Flowers, } \\
\text { Whole plant }\end{array}$ & $\begin{array}{l}\text { Leaves and flower } \\
\text { *Paste (wounds) } \\
\text { Whole plant } \\
\text { *Juice (diarrhea and stomach problem) }\end{array}$ & 0.34 & 0.26 \\
\hline Cirsium arvense $\mathrm{L}$. & $\begin{array}{l}\text { Stems, } \\
\text { Roots }\end{array}$ & $\begin{array}{l}\text { Stems } \\
\text { *Chewing (toothache) } \\
\text { Roots } \\
\text { *Ash (wounds) }\end{array}$ & 0.16 & 0.16 \\
\hline Conyza ambigua $\mathrm{L}$. & $\begin{array}{l}\text { Roots, } \\
\text { Whole plant }\end{array}$ & $\begin{array}{l}\text { Roots } \\
\text { *Root tea (menstrual disorder) } \\
\text { Whole plant } \\
\text { *Tea (alcoholic poisoning) } \\
\text { *Paste (piles) } \\
\text { *Steam (enhance sneezing during cold) }\end{array}$ & 0.18 & 0.14 \\
\hline Eclipta alba $\mathrm{L}$. & Leaves & $\begin{array}{l}* \text { Chewing few leaves daily (eye health) } \\
* \text { Extract (high blood pressure) }\end{array}$ & 0.13 & 0.24 \\
\hline $\begin{array}{l}\text { Parthenium } \\
\text { hysterophorus L. }\end{array}$ & Whole plant & $\begin{array}{l}* \text { Decoction (diarrhea, urinary infection, fever } \\
\text { and malaria) }\end{array}$ & 0.20 & 0.31 \\
\hline Sonchus asper L. & Whole plant & *Ash (wounds) & 0.11 & 0.17 \\
\hline $\begin{array}{l}\text { Sonchus oleraceus } \\
\text { L. }\end{array}$ & Leaves & $\begin{array}{l}\text { *Juice (eye disease) } \\
\text { *Paste (inflammation) }\end{array}$ & 0.23 & 0.13 \\
\hline $\begin{array}{l}\text { Cordia dichotoma } \\
\text { G. Forst }\end{array}$ & $\begin{array}{l}\text { Seeds, } \\
\text { Barks, } \\
\text { Fruits, } \\
\text { Leaves }\end{array}$ & $\begin{array}{l}\text { Seeds } \\
\text { *Powder apply on skin allergy } \\
\text { Barks } \\
\text { *Paste (swelling) } \\
\text { *Maswak (teeth disease and headache) } \\
\text { Fruits } \\
\text { *Paste (skin allergy) } \\
\text { Leaves } \\
\text { *Juice (cooling) }\end{array}$ & 0.35 & 0.47 \\
\hline
\end{tabular}


European Journal of Biology

ISSN 2520-4738 (Online)

Vol.5, Issue 1, pp 41-62, 2020

www.ajpojournals.org

\begin{tabular}{|c|c|c|c|c|}
\hline $\begin{array}{l}\text { Heliotropium } \\
\text { indicum } \mathrm{L} \text {. }\end{array}$ & $\begin{array}{l}\text { Leaves, } \\
\text { Whole plant }\end{array}$ & $\begin{array}{l}\text { Leaves } \\
\text { *Infusion (asthma) } \\
\text { *Paste (insect sting) } \\
\text { Whole plant } \\
\text { *Decoction (thrush and diabetes) } \\
\text { *Boiled and rubbed on heat rash }\end{array}$ & 0.37 & 0.18 \\
\hline $\begin{array}{l}\text { Chenopodium } \\
\text { berlandieri Moq. }\end{array}$ & Whole plant & *Cooked (gout, kidney stone and arthritis) & 0.30 & 0.47 \\
\hline $\begin{array}{l}\text { Chenopodiastrum } \\
\text { murale L. }\end{array}$ & Seeds & $\begin{array}{l}\text { *Powder (making bread with wheat and } \\
\text { kidney stone }\end{array}$ & 0.41 & 0.68 \\
\hline
\end{tabular}

Contin......

\begin{tabular}{|c|c|c|c|c|}
\hline Species name & Part used & Recipes with ailments & $\overline{\mathbf{U V}}$ & RFC \\
\hline Sisymbrium irio L. & $\begin{array}{l}\text { Leaves, } \\
\text { Seeds, } \\
\text { Whole plant }\end{array}$ & $\begin{array}{l}\text { Leaves } \\
\text { *Infusion (throat and chest infection) } \\
\text { Seeds } \\
\text { *Seed in water (asthma) } \\
\text { Whole plant } \\
\text { *Paste (inflammation) }\end{array}$ & 0.59 & 0.19 \\
\hline Cleome viscosa $\mathrm{L}$. & $\begin{array}{l}\text { Leaves, } \\
\text { Whole plant }\end{array}$ & $\begin{array}{l}\text { Leaves } \\
\text { *Paste (wounds) } \\
\text { Whole plant } \\
\text { *Juice (stimulate the appetite) } \\
\text { *Decoction (improve the digestive system and } \\
\text { enhance hunger) }\end{array}$ & 0.22 & 0.11 \\
\hline $\begin{array}{l}\text { Chenopodium } \\
\text { album } \mathrm{L} \text {. }\end{array}$ & $\begin{array}{l}\text { Leaves, } \\
\text { Seeds, } \\
\text { Stems, } \\
\text { Roots, } \\
\text { Whole plant }\end{array}$ & $\begin{array}{l}\text { Leaves } \\
\text { *Paste (swelling) } \\
\text { Seeds } \\
\text { *Chewing (urinary infection) } \\
\text { Stems } \\
\text { *Juice (freckles) } \\
\text { Roots } \\
\text { *Juice (blood dysentery) } \\
\text { Whole plant } \\
\text { *Decoction (sunstroke and teeth disease) }\end{array}$ & 0.68 & 0.87 \\
\hline $\begin{array}{l}\text { Chenopodium } \\
\text { berlandieri Moq. }\end{array}$ & Whole plant & *Cooked (gout, kidney stone and arthritis) & 0.30 & 0.47 \\
\hline $\begin{array}{l}\text { Chenopodiastrum } \\
\text { murale L. }\end{array}$ & Seeds & $\begin{array}{l}\text { *Powder (making bread with wheat and } \\
\text { kidney stone }\end{array}$ & 0.41 & 0.68 \\
\hline $\begin{array}{l}\text { Convolvulus } \\
\text { arvensis } \mathrm{L} .\end{array}$ & $\begin{array}{l}\text { Flower, } \\
\text { Leaves, }\end{array}$ & $\begin{array}{l}\text { Flower } \\
\text { *Tea (fever) }\end{array}$ & 0.23 & 0.12 \\
\hline
\end{tabular}


European Journal of Biology

ISSN 2520-4738 (Online)

Vol.5, Issue 1, pp 41-62, 2020

www.ajpojournals.org

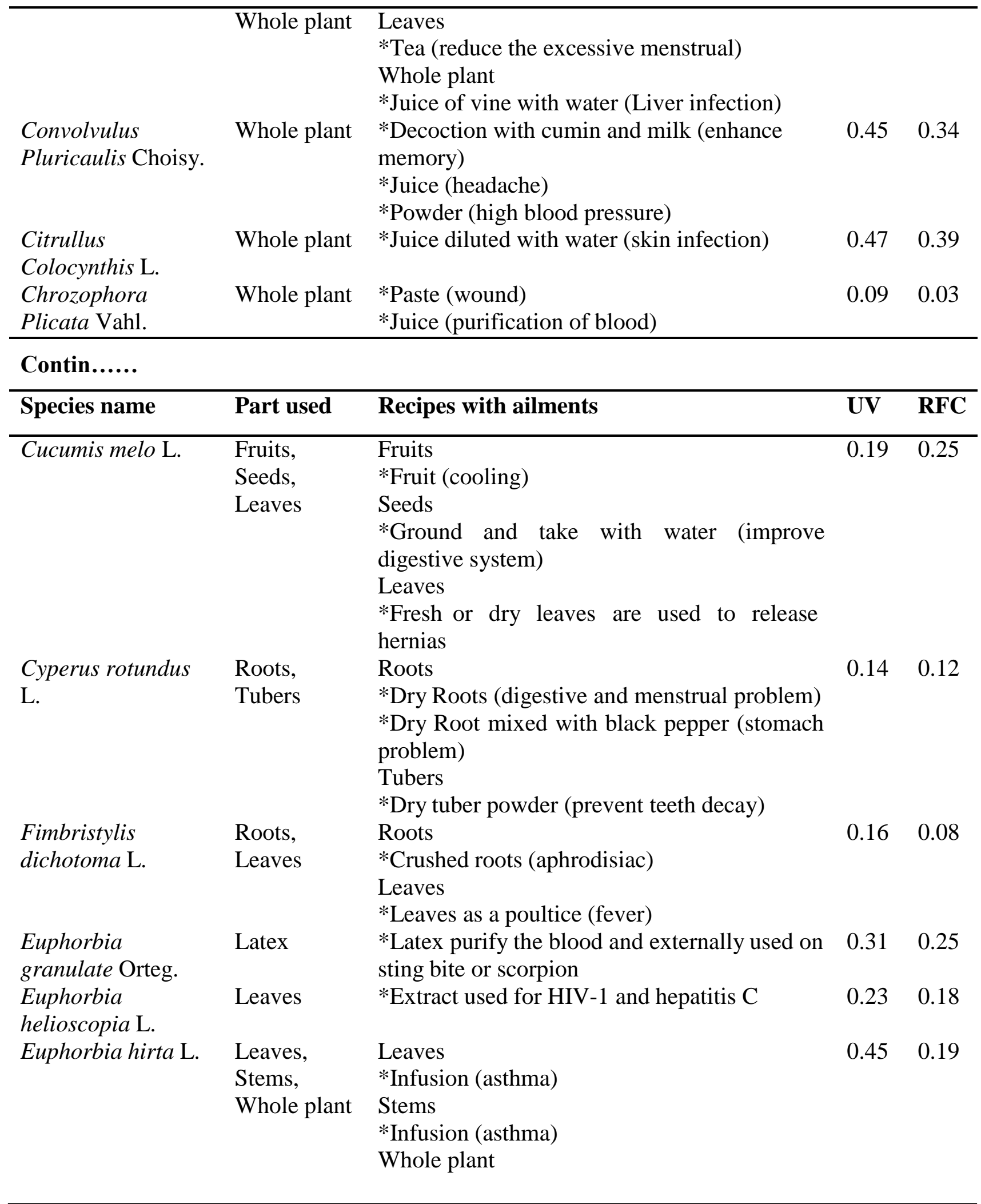


European Journal of Biology

ISSN 2520-4738 (Online)

Vol.5, Issue 1, pp 41-62, 2020

www.ajpojournals.org

\begin{tabular}{|c|c|c|c|c|}
\hline & & $\begin{array}{l}\text { *Paste (sting bite, skin infection, inflammation } \\
\text { and fungal infection) } \\
\text { *Decoction (foot athletes and fungal infection) }\end{array}$ & & \\
\hline $\begin{array}{l}\text { Euphorbia } \\
\text { microphylla Heyne } \\
\text { ex. Roth. }\end{array}$ & Whole plant & $\begin{array}{l}\text { *Infusion (asthma) } \\
\text { *Decoction (improve digestive system) }\end{array}$ & 0.17 & 0.21 \\
\hline $\begin{array}{l}\text { Euphorbia } \\
\text { thymifolia } \mathrm{L} .\end{array}$ & $\begin{array}{l}\text { Leaves, } \\
\text { Whole plant }\end{array}$ & $\begin{array}{l}\text { Leaves } \\
\text { *Decoction (kidney disease) } \\
\text { *Paste (headache) } \\
\text { Whole plant } \\
\text { *Decoction (diarrhea, lung problem, eye wash } \\
\text { and veneral diseases) }\end{array}$ & 0.19 & 0.08 \\
\hline $\begin{array}{l}\text { Hydrilla verticillata } \\
\text { (L.f.) Royle }\end{array}$ & Whole plant & $\begin{array}{l}\text { * Chewing or juice (Nervous system disorder, } \\
\text { build blood cells and regeneration of skin) }\end{array}$ & 0.23 & 0.09 \\
\hline
\end{tabular}

Contin......

\begin{tabular}{|c|c|c|c|c|}
\hline Species name & Part used & Recipes with ailments & $\mathbf{U V}$ & RFC \\
\hline $\begin{array}{l}\text { Ricinus communis } \\
\text { L. }\end{array}$ & Fruits & $\begin{array}{l}\text { Oil } \\
* \text { Castor oil helpful for reducing constipation } \\
\text { when taken by mouth } \\
* \text { Dry eyes cure by castor oil } \\
* \text { Castor oil removed the dandruff in hair } \\
* \text { Few drops of castor oil used for better vision }\end{array}$ & 0.54 & 0.89 \\
\hline $\begin{array}{l}\text { Acacia } \\
\text { Hayne. }\end{array}$ & $\begin{array}{l}\text { Stems, } \\
\text { Whole plant }\end{array}$ & $\begin{array}{l}\text { Stems } \\
\text { *Extract (loose motion, diarrhea and urinary } \\
\text { bladder pain) } \\
\text { *Chewing (oral ailment) } \\
\text { Whole plant } \\
\text { *Whole plant juice used for wound washing }\end{array}$ & 0.66 & 0.93 \\
\hline $\begin{array}{l}\text { Oxalis corniculata } \\
\text { L. }\end{array}$ & $\begin{array}{l}\text { Leaves, } \\
\text { Whole plant }\end{array}$ & $\begin{array}{l}\text { Whole plant } \\
\text { *Juice (stomach, influenza, urinary disease, } \\
\text { insect bite and scurvy } \\
\text { Leaves } \\
\text { *Juice (insect sting and skin cramps) }\end{array}$ & & \\
\hline Accaia nilotica $\mathrm{L}$. & $\begin{array}{l}\text { Barks, } \\
\text { Flowers, } \\
\text { Leaves, } \\
\text { Seeds, } \\
\text { Whole plant }\end{array}$ & $\begin{array}{l}\text { Barks } \\
* \text { Chewing (reduce the teeth lose and stop the } \\
\text { bleeding) } \\
* \text { Gums (skin irritation, inflammation and } \\
\text { Diabetes) } \\
\text { *Powder (toothaches) } \\
\text { *Boiled with water and wash the wounds } \\
\text { Flowers } \\
\text { *Fresh (loose motion) }\end{array}$ & 0.78 & 0.95 \\
\hline
\end{tabular}


European Journal of Biology

ISSN 2520-4738 (Online)

Vol.5, Issue 1, pp 41-62, 2020

www.ajpojournals.org

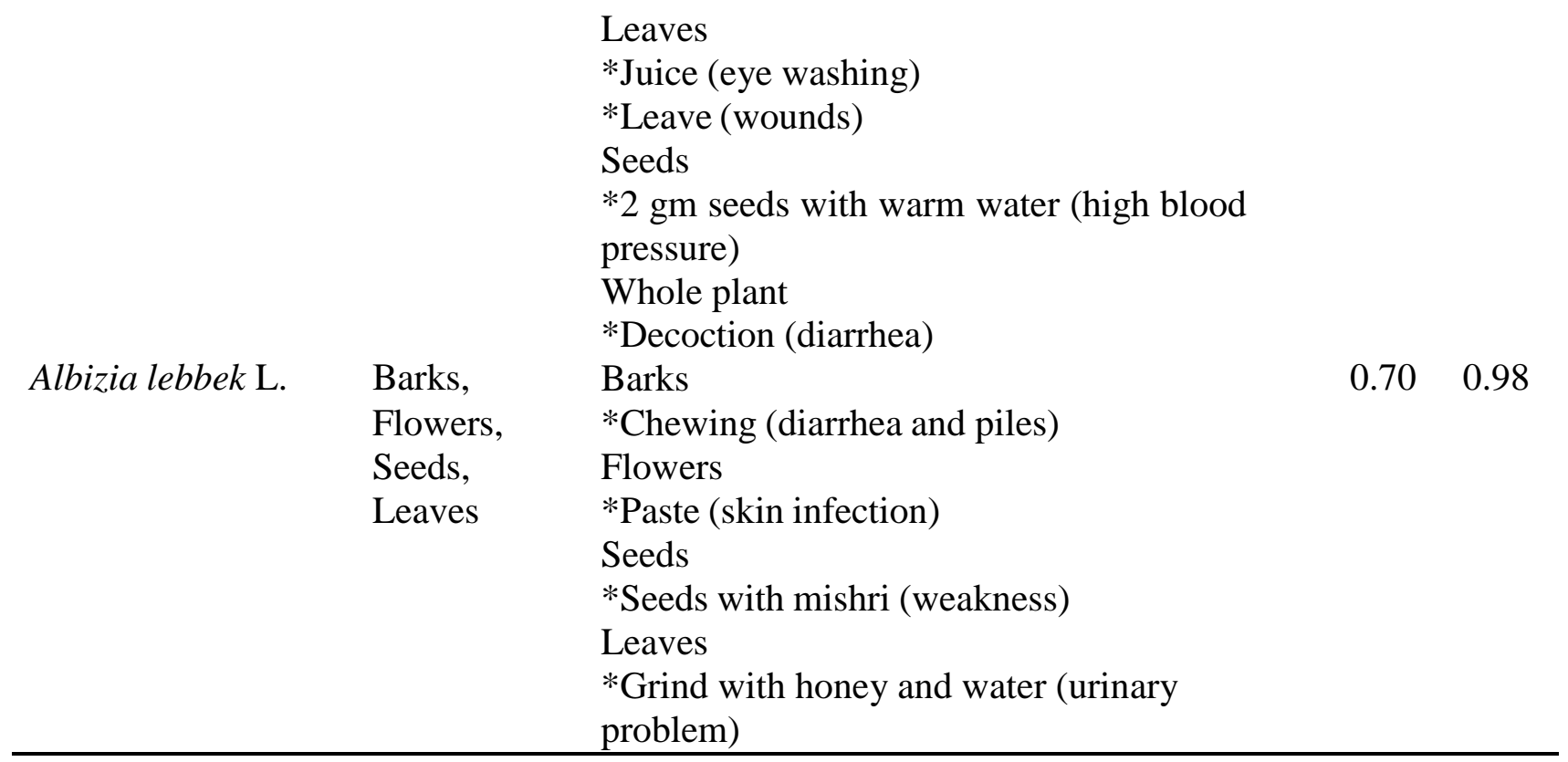

\section{Contin......}

\begin{tabular}{|c|c|c|c|c|}
\hline Species name & Part used & Recipes with ailments & $\mathbf{U V}$ & RFC \\
\hline Cassia fistula L. & $\begin{array}{l}\text { Leaves, } \\
\text { Pods, Barks }\end{array}$ & $\begin{array}{l}\text { Leaves } \\
* \text { Extract (skin pathogen) } \\
\text { Pods } \\
\text { *Decoction (malaria, diabetes and kidney } \\
\text { stone) } \\
\text { Bark } \\
\text { *Paste (skin disease) } \\
\text { *Decoction (washing wounds) }\end{array}$ & 0.61 & 0.84 \\
\hline $\begin{array}{l}\text { Ocimum } \\
\text { Basilicum L. }\end{array}$ & $\begin{array}{l}\text { Leaves, } \\
\text { Seeds, } \\
\text { Whole plant }\end{array}$ & $\begin{array}{l}\text { Leaves } \\
\text { *Juice (Influenza and enhance digestion) } \\
\text { *Paste (skin allergy and snake sting) } \\
\text { Seeds } \\
\text { *Infusion (diarrhea) } \\
\text { Whole plant } \\
\text { *Juice (digestive and nervous system disorder) }\end{array}$ & 0.45 & 0.59 \\
\hline Lathyrus aphaca L. & Seeds & *Chewing (teeth diseases) & 0.19 & 0.09 \\
\hline $\begin{array}{l}\text { Pongamia pinnata } \\
\text { L. }\end{array}$ & $\begin{array}{l}\text { Seeds, } \\
\text { Leaves, } \\
\text { Stems, } \\
\text { Roots }\end{array}$ & $\begin{array}{l}\text { Seed } \\
* \text { Oil stomach and liver disease } \\
\text { *Paste joint disorder and sores } \\
\text { Leaves } \\
\text { *Decoction (cough) } \\
\text { *Paste (skin infection and stop bleeding) }\end{array}$ & 0.44 & 0.81 \\
\hline
\end{tabular}




\begin{tabular}{|c|c|c|c|c|}
\hline & & $\begin{array}{l}\text { Stems } \\
\text { *Maswak (Stop spleen enlargement) } \\
\text { Roots } \\
\text { *Juice (ulcer and toothaches) }\end{array}$ & & \\
\hline Phyllanthus niruri & Roots, & Roots & 0.18 & 0.08 \\
\hline & Leaves & $\begin{array}{l}\text { *Juice (bladder stone) } \\
\text { *Infusion (chronic dysentery and cold) } \\
\text { Leaves } \\
\text { *Infusion (dysentery, stomach problem and } \\
\text { cold) }\end{array}$ & & \\
\hline Avena fatua $\mathrm{L}$. & Seeds & $\begin{array}{l}\text { *Paste mixed with salt (wounds) } \\
\text { *Seeds are used as a cereal for nourishment }\end{array}$ & 0.30 & 0.45 \\
\hline $\begin{array}{l}\text { Brachiaria ramose } \\
\mathrm{L} .\end{array}$ & $\begin{array}{l}\text { Roots, } \\
\text { Whole plant }\end{array}$ & $\begin{array}{l}\text { Roots } \\
\text { *Paste (kidney pain) } \\
\text { Whole plant } \\
\text { *Ash( insect sting) }\end{array}$ & 0.23 & 0.18 \\
\hline Cenchrus ciliaris L. & Whole plant & $\begin{array}{l}\text { *Juice (kidney problem and tumor) } \\
\text { *Paste (wound) }\end{array}$ & 0.15 & 0.12 \\
\hline \multicolumn{5}{|l|}{ Contin...... } \\
\hline Species name & Part used & Recipes with ailments & $\mathbf{U V}$ & RFC \\
\hline $\begin{array}{l}\text { Cyanodon dactylon } \\
\text { L. }\end{array}$ & $\begin{array}{l}\text { Leaves, } \\
\text { Whole plant }\end{array}$ & $\begin{array}{l}\text { Leaves } \\
\text { *Paste (wound) } \\
\text { Whole plant } \\
\text { *Decoction (cancer, cough, headache, dropsy, } \\
\text { stone, snake sting, wound and toothaches) }\end{array}$ & 0.37 & 0.15 \\
\hline $\begin{array}{l}\text { Dactyloctenum } \\
\text { aegyptium } \mathrm{L} .\end{array}$ & Leaves & $\begin{array}{l}\text { *Infusion (accelerate childbirth) } \\
\text { *Decoction (dysentery) }\end{array}$ & 0.29 & 0.17 \\
\hline $\begin{array}{l}\text { Demostachya } \\
\text { bipinnata L. }\end{array}$ & Whole plant & *Decoction (dysentery) & 0.12 & 0.14 \\
\hline $\begin{array}{l}\text { Echinochloa colona } \\
\text { L. }\end{array}$ & Stems & *Paste (wounds) & 0.16 & 0.13 \\
\hline $\begin{array}{l}\text { Echinochola } \\
\text { crusgalli L. }\end{array}$ & Roots & *Paste (wounds) & 0.18 & 0.07 \\
\hline $\begin{array}{l}\text { Imperata cylindrica } \\
\mathrm{L} .\end{array}$ & $\begin{array}{l}\text { Flowers, } \\
\text { Roots, } \\
\text { Whole plant }\end{array}$ & $\begin{array}{l}\text { Flowers } \\
* \text { Paste (wound)s } \\
\text { *Decoction (urinary infection and fever) } \\
\text { Roots } \\
\text { *Decoction (digestive system diseases) } \\
\text { Whole plant } \\
\text { *Extract (cancer) }\end{array}$ & 0.10 & 0.03 \\
\hline & Whole plant & *Infusion (heart problem) & 0.23 & 0.05 \\
\hline
\end{tabular}


European Journal of Biology

ISSN 2520-4738 (Online)

Vol.5, Issue 1, pp 41-62, 2020

www.ajpojournals.org

\begin{tabular}{|c|c|c|c|c|}
\hline $\begin{array}{l}\text { Sachharum } \\
\text { benghalense Retz. }\end{array}$ & $\begin{array}{l}\text { Leaves, } \\
\text { Stems }\end{array}$ & $\begin{array}{l}\text { Leaves } \\
\text { *Juice (eye wash) } \\
\text { *Decoction (urinary infection) } \\
\text { Stems } \\
\text { *Juice (sore throat) }\end{array}$ & 0.15 & 0.17 \\
\hline Rumex dentatus L. & $\begin{array}{l}\text { Roots, } \\
\text { Leaves, } \\
\text { Whole plant }\end{array}$ & $\begin{array}{l}\text { Roots } \\
\text { *Decoction (stomach problem and intestinal } \\
\text { parasite } \\
\text { *Paste (swelling) } \\
\text { Leaves } \\
\text { *Juice (headache) } \\
\text { *Paste (wounds) } \\
\text { Whole plant } \\
\text { *Decoction (reduce body pain) }\end{array}$ & 0.22 & 0.23 \\
\hline $\begin{array}{l}\text { Portulaca oleracea } \\
\text { L. }\end{array}$ & $\begin{array}{l}\text { Leaves } \\
\text { Whole plant }\end{array}$ & $\begin{array}{l}\text { Leaves } \\
\text { *Juice (heart disease, cough and earaches) } \\
\text { *Tea (stomach disease and headache) } \\
\text { Whole plant } \\
\text { *Extract (muscle relaxation and wounds) } \\
\text { *Juice (skin diseases and insect bite) }\end{array}$ & 0.34 & 0.25 \\
\hline
\end{tabular}

Contin......

\begin{tabular}{|c|c|c|c|c|}
\hline Species name & Part used & Recipes with ailments & $\mathbf{U V}$ & RFC \\
\hline $\begin{array}{l}\text { Anagallis arvensis } \\
\text { L. }\end{array}$ & Whole plant & $\begin{array}{l}\text { *Infusion (skin diseases and liver diseases) } \\
\text { *Paste (skin itches and warts) }\end{array}$ & 0.16 & 0.16 \\
\hline $\begin{array}{l}\text { Renunculus } \\
\text { muricatus L. }\end{array}$ & Fruits & *Fresh or juice (asthma and fever) & 0.28 & 0.26 \\
\hline Dhatura alba L. & $\begin{array}{l}\text { Leaves, } \\
\text { Seeds }\end{array}$ & $\begin{array}{l}\text { Leaves } \\
\text { *Paste of roasted leaves (reduce full body } \\
\text { pain) } \\
\text { *Smoke (asthma) } \\
\text { *Juice (ear disease) } \\
\text { Seeds } \\
\text { *Oil (stimulate hair growth) }\end{array}$ & 0.10 & 0.04 \\
\hline Physalis minima $\mathrm{L}$. & $\begin{array}{l}\text { Fruits, } \\
\text { Leaves, } \\
\text { Roots, } \\
\text { whole plant }\end{array}$ & $\begin{array}{l}\text { Fruits } \\
\text { *Fresh (enhance appetite) } \\
\text { Leaves } \\
\text { *Paste (headache and skin rash) } \\
\text { Roots } \\
\text { *Extract (fever) } \\
\text { *Decoction (diabetes) }\end{array}$ & 0.21 & 0.14 \\
\hline
\end{tabular}


European Journal of Biology

ISSN 2520-4738 (Online)

Vol.5, Issue 1, pp 41-62, 2020

www.ajpojournals.org

\begin{tabular}{|c|c|c|c|c|}
\hline & & $\begin{array}{l}\text { Whole plant } \\
\text { *Extract (cancer) }\end{array}$ & & \\
\hline $\begin{array}{l}\text { Withania somnifera } \\
\text { L. }\end{array}$ & Whole plant & $\begin{array}{l}\text { *Juice (tiredness. High sugar level in blood } \\
\text { and high cholesterol level) }\end{array}$ & & \\
\hline \multirow[t]{5}{*}{ Solanum nigrum L. } & $\begin{array}{l}\text { Fruits, } \\
\text { Whole plant }\end{array}$ & $\begin{array}{l}\text { Fruits } \\
* \text { Juice (teeth diseases) }\end{array}$ & \multirow[t]{5}{*}{0.47} & \multirow[t]{5}{*}{0.57} \\
\hline & & *Fresh (eye disease and fever) & & \\
\hline & & *Paste (headaches and skin allergy) & & \\
\hline & & Whole plant & & \\
\hline & & *Paste ( wounds) & & \\
\hline \multirow{6}{*}{$\begin{array}{l}\text { Sphenoclea } \\
\text { zeylanica Gaertn. } \\
\text { Phyla nodiflora L. }\end{array}$} & Leaves & *Paste (sting of poisonous animals) & 0.17 & 0.19 \\
\hline & Leaves, & Leaves & \multirow[t]{5}{*}{0.22} & \multirow[t]{5}{*}{0.33} \\
\hline & Whole plant & *3-4 fresh leaves (piles) & & \\
\hline & & *Paste (infected skin by ulcer) & & \\
\hline & & Whole plant & & \\
\hline & & *Whole plant juice (cough and fever) & & \\
\hline
\end{tabular}

Part used: The Ethnobotanical data related to part used of 70 species was displayed because 11 plant species had no Ethnobotanical data and $57 \%$ whole plant was used in the ayurvedic field followed by leaves $(53 \%)$ and roots $(26 \%)$. According to the data cited by the informants, the \%age of using pods (Cassia fistula L.), tubers (Cyperus rotundus L.) and latex (Euphorbia granulate Orteg.) was minimal because they were used only by one plant. The use of leaves other than other plant plants in the medicinal field was good because leaves are easily collected and sustainable method in ecology with no fear of extinction [7].

Table 6: Percentage of plant part used

\begin{tabular}{llllll}
\hline Part used & $\begin{array}{l}\text { No. of } \\
\text { Species }\end{array}$ & Percentage & Part used & $\begin{array}{l}\text { No. of } \\
\text { Species }\end{array}$ & Percentage \\
\hline Leaves & 37 & 53 & Roots & 18 & 26 \\
Seeds & 16 & 23 & Fruits & 6 & 8 \\
Flowers & 8 & 11 & Stems & 8 & 11 \\
Barks & 5 & 7 & Whole plant & 40 & 57 \\
\hline
\end{tabular}

Informant consensus factor (ICF): ICF was used to focus on the consistency of data related to particular disease categories. The disease was divided into 15 categories based on the recorded ailments by participants. The range of ICF was recorded from 0.45 to 0.78 . The lowest ICF value was observed in hormonal disorder $(0.45)$ and the highest value was shown by respiratory disease (0.78). The highest ICF value predicts that the high \%age of participants was used plants 
European Journal of Biology

ISSN 2520-4738 (Online)

Vol.5, Issue 1, pp 41-62, 2020

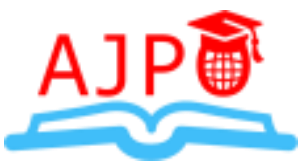

www.ajpojournals.org

for an illness of a comprehensive category. The local people of Haroonabad were used Euphorbia helioscopia L. leaves to cure HIV and hepatitis C and Withiana somnifera L. was used to treat the high sugar and cholesterol level. The leaves of Oxalis corniculata L. were used to treat the scurvy ailment and Cynodon dactylon L. was used in cancer treatment.

Table 7: ICF of recorded plant species with respect to different ailments.

\begin{tabular}{lllllll}
\hline $\begin{array}{l}\text { Sr. } \\
\text { No. }\end{array}$ & Disease category & $\begin{array}{l}\text { No. of use } \\
\text { reports }\end{array}$ & $\begin{array}{l}\text { Percentage } \\
\text { use reports }\end{array}$ & $\begin{array}{l}\text { No. of } \\
\text { species }\end{array}$ & $\begin{array}{l}\text { Percentage } \\
\text { of use species }\end{array}$ & *ICF \\
\hline 1 & Respiratory diseases & 41 & 48 & 10 & 12 & 0.78 \\
2 & Nervous system disorder & 7 & 8 & 4 & 5 & 0.50 \\
3 & Skin infection & 61 & 72 & 24 & 30 & 0.61 \\
4 & Urinary diseases & 19 & 22 & 10 & 12 & 0.50 \\
5 & Hormonal disorder & 10 & 12 & 6 & 7 & 0.45 \\
6 & Heart diseases & 11 & 13 & 5 & 6 & 0.60 \\
7 & Digestive system diseases & 17 & 20 & 9 & 11 & 0.50 \\
8 & Stomach diseases & 43 & 51 & 11 & 14 & 0.76 \\
9 & Liver diseases & 15 & 18 & 3 & 3 & 0.85 \\
10 & Wounds & 70 & 82 & 18 & 22 & 0.75 \\
11 & Insect sting & 31 & 36 & 11 & 14 & 0.67 \\
12 & Muscle disorder & 13 & 15 & 3 & 3 & 0.83 \\
13 & Stone & 27 & 32 & 7 & 9 & 0.77 \\
14 & Diabetes & 9 & 11 & 4 & 5 & 0.63 \\
15 & Eye, ear, throat and & 29 & 34 & 13 & 16 & 0.57 \\
& mouth disease & & & & & \\
\hline
\end{tabular}

\section{CONCLUSION}

The biodiversity of any area indicates the importance of the area. Poaceae and Therophyte species were more prominent in the study area. Wild flora maintains the ecosystem and sustainability in the environment and they also had a major role in the medical field. Present research discovered the medicinal plants that mostly used by local inhabitants to cure various ailments. It tries to attract the attention towards the conservation policy of wild plants. The wild plants in Haroonabad have very importance in the homeopathic medical field but biodiversity was not high.

\section{RECOMMENDATIONS}

1. The native people of studied region have used traditional botanical knowledge to heal the various ailments but the accessibility of plants is atypical. Consequently, the traditional healers and the native people should be awake on how to use plants for a variety of objectives and the traditional healers should cultivate some plants in their home gardens.

2. There are some medicinal plants used to treat different diseases. But the chemical components of these plants are not well known. Therefore, research on chemical analysis of these plants should be performed.

3. There is a need to authorize the indigenous communities and make sure their active participation in sustainable harvesting and conservation of natural resources. Different 
European Journal of Biology

ISSN 2520-4738 (Online)

Vol.5, Issue 1, pp 41-62, 2020

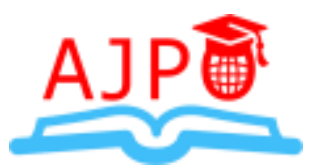

www.ajpojournals.org

universities should collaborate with indigenous communities and recognize them as 'knowledge site' on a particular subject to uphold their status and conserve its knowledge.

\section{REFERENCES}

1. Anonymous, 2009. Flora of Ziarat: Ethnobotanic and Medicinal Importance, pp. 71.

2. Anyinam C. (1995). "Ecology and Ethnomedicine: Exploring Links between Current Environment Crisis and Indigenous Me- dical Practices," Soc. Sci. Med., 40(3): 321-329.

3. Barla A, Bİrman H, Kültür Ş and Öksüz S. (2006). Secondary metabolites from Euphorbia helioscopia and their vasodepressor activity. Turk. J. Chem. 30(3): 325-332.

4. Dike IP, Obembe OO and Adebiyi FE. (2012). Ethnobotanical survey for potential antimalarial plants in south-western Nigeria. J. Ethnopharmacol., 144(3): 618-626.

5. Faiz A,Ghufarn MA, Mian A and Akhtar T. (2014). Floral diversity of Tolipir National Park (TNP), Azad Jammu and Kashmir. Biologia, 60(1): 43-55.

6. Ghimire SK, Gimenez O, Pradel R, McKey D and Aumeeruddy-Thomas Y. (2008). Demographic variation and population viability in a threatened Himalayan medicinal and aromatic herb Nardostachys grandiflora: matrix modelling of harvesting effects in two contrasting habitats. J. Appl. Ecol., 45(1): 41-51.

7. Giday M, Asfaw Z and Woldu Z. (2010). Ethnomedicinal study of plants used by Sheko ethnic group of Ethiopia. J. Ethnopharmacol., 132 (1): 75-85.

8. Heinrich M, Ankli A, Frei B, Weimann C and Sticher O. (1998). Medicinal plants in Mexico: Healers' consensus and cultural importance. Soc. Sci. Med., 47(11): 1859-1871.

9. Ilyas M, Qureshi R, Shaheen H, Ahmad W and Munir M. (2014). Phytodiversity and plant life of Khanpur Dam, Khyber Pakhtunkhwa, Pakistan. Pak. J. Bot., 46(3): 841-849.

10. Jima TT and Megersa M. (2018). Ethnobotanical study of medicinal plants used to treat human diseases in berbere district, bale zone of oromia regional state, south east Ethiopia. Evid.-Based Complementary Altern. Med., pp 1-16.

11. Khan AM, Qureshi R, Qaseem MF, Munir M, Ilyas M and Saqib Z. (2015). Floristic checklist of district kotli, azad jammu and kashmir. Pak. J. Bot., 47(5): 1957-1968.

12. Lee S, Xiao C and Pei S. (2008). Ethnobotanical survey of medicinal plants at periodic markets of Honghe Prefecture in Yunnan Province, SW China. J. Ethnopharmacol., 117(2): 362-377.

13. Noman A. (2003). Influence of different doses of nitrogen fertilizer on ajwain. M.Sc. Dissertation. pp-1. Univ. of Agric. Faisalabad. Pakistan.

14. Phillips O, Gentry AH, Reynel C, Wilkin P, Galvez-Durand B. (1994). Quantitative ethnobotany and Amazonian conservation. Conserv. Biol., 8(1): 225-248.

15. Shad AA, Shah HU and Bakht J. (2013). Ethnobotanical assessment and nutritive potential of wild food plants. J. Anim. Plant Sci., 23(1): 92-99.

16. Shah A, Poudel RC, Ishtiaq M, Sarvat R, Shahzad H, Abbas A, Shoaib S, Nuzhat R, Noor UD, Mahmooda H, Summaya A, Ifra and Ihsan U. (2019). Ethnobotanical study of medicinal plants of namal valley, salt range, Pakistan. Appl. Ecol. Environ. Res., 17(2):4725-4805. 
European Journal of Biology

ISSN 2520-4738 (Online)

Vol.5, Issue 1, pp 41-62, 2020

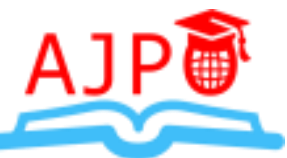

www.ajpojournals.org

17. Shinwari ZK and Qaiser M. (2011). Efforts on conservation and sustainable use of medicinal plants of Pakistan. Pak. J. Bot., 43(1): 5-10.

18. Vitalini S, Iriti M, Puricelli C, Ciuchi D, Segale A and Fico G. (2013). Traditional knowledge on medicinal and food plants used in Val San Giacomo (Sondrio, Italy) -An alpine ethnobotanical study. J. Ethnopharmacol., 145(2): 517-529. 